\title{
Identificación de atractivos y recursos turísticos como reactivación del destino bajo los efectos del covid19, Ecuador ${ }^{1}$
}

\section{Identification of tourist attractions and resources as a reactivation of the destination under the effects of covid19, Ecuador}

DOI: $10.46932 / \mathrm{sfjdv} 2 \mathrm{n} 4-027$

Received in: March 1st, 2021

Accepted in: May 30th, 2021

\section{Ing. Brenda Cecilia Otero Navarrete}

Ingeniera en Marketing, cursante del programa de maestría de investigación en estadística aplicada de la Universidad Técnica de Manabí, Ecuador.

E-mail: brendaotero1994@ outlook.es

\section{Mr.C. Luis Daniel Zambrano Molina}

Docente de la Uleam, Extensión Bahía y coordinador de la carrera de Administración de Empresas.

E-mail: luis.zambrano@uleam.edu.ec

\section{Dr. C. Frank Ángel Lemoine Quintero}

Docente titular de la carrera de Mercadotecnia en la Universidad Laica Eloy Alfaro de Manabí. Extensión Bahía de Caráquez. Investigador acreditado por la SENESCYT. Provincia Manabí, Ecuador. E-mail: fangel64@gmail.com

\section{Dr.C Norma Rafaela Hernández Rodríguez}

Docente de la Universidad de Oriente, Investigadora acreditada de la facultad de Ciencias Económicas y Empresariales. Doctora en Ciencias Económicas y Empresariales. Cuba,

E-mail: norma@uo.edu.cu

\section{RESUMEN}

La investigación se realizó con la finalidad de recopilar información del estado de conservación de atractivos y recursos turísticos existente en el cantón Sucre con la finalidad de identificarlo y reconocerle de acuerdo a indicadores y variables orientadas por el MINTUR con el propósito de fortalecer la actividad turística y económica en la zona objeto estudio bajo los efectos del Covid19. El método de observación permitió analizar minuciosamente cada aspecto físico del atractivo y su estado de preservación. El arte de la ciencia bibliográfica, revistas, artículo referente al tema aportó con datos que tributaron significativamente a la verificación de cada uno de estos bajo las condiciones pandémicas y a su abandono por confinamiento. La caracterización de los mismo permitió reconocer las especificaciones, los puntos fuertes y potencialidades para desarrollar un turismo sostenible en el destino Sucre. Se concluye atribuyéndole al estudio el desarrollo de estrategias publicitarias que potencialicen cada atractivo y recurso turísticos como factor contribuyente de la actividad económica en la zona.

Palabras claves: Atractivos turísticos, recursos turísticos, reactivación turística, servicios.

\footnotetext{
${ }^{1}$ Proyecto de Investigación: Observatorio turístico para el control de la gestión del destino turístico Sucre-San Vicente, Jama y Pedernales
} 


\begin{abstract}
The research was carried out in order to collect information on the state of conservation of tourist attractions and resources existing in the Sucre canton in order to identify and recognize it according to indicators and variables guided by the MINTUR in order to strengthen tourism activity and economic in the area under study under the effects of Covid19. The observation method allowed a detailed analysis of each physical aspect of the attraction and its state of preservation. The art of bibliographic science, magazines, an article on the subject contributed data that contributed significantly to the verification of each of these under pandemic conditions and their abandonment due to confinement. The characterization of the same allowed to recognize the specifications, the strong points and potentialities to develop a sustainable tourism in the Sucre destination. It is concluded by attributing to the study the development of advertising strategies that enhance each tourist attraction and resource as a contributing factor of economic activity in the area.
\end{abstract}

Keywords: Tourist attractions, tourist resources, tourist reactivation, services

\title{
1 INTRODUCCIÓN
}

En la actualidad uno de los problemas que afecta al desarrollo turístico en el cantón Sucre al Noreste de la provincia de Manabí es la falta de información y promoción de sus recursos y atractivos turísticos, por tal motivo es una actividad no muy desarrollada, el cual debe ser de mayor importancia tanto para el municipio, asociaciones, a causa de que el turismo promueve economía mejorando el estilo de vida de las personas. El cantón Sucre se encuentra ubicado al Nor-este de la provincia de Manabí, tiene una población de 57.159 habitantes. El 21 de octubre de 1867 se da la primera creación del cantón y el 3 de noviembre de 1875 se legaliza definitivamente y se suscribe la primera acta constitutiva. Su cabecera cantonal es la ciudad de Bahía de Caráquez.

Según (Rivera S. C., 2009) atractivo turístico se define como el conjunto de elementos materiales que son susceptibles de ser transformados en un producto turístico que tenga capacidad para incidir sobre el proceso decisión del turista, pero (Corbuse-turismo, 2011) argumenta que los atractivos turísticos son aquellas características y puntos de interés para los turistas que llaman su atención y atrae a los viajeros.

Es una actividad donde el turista disfruta de un espacio libre, no tiene preocupación y puede disfrutar de las diferentes actividades recreativas existente en la zona, actividades como los deportes acuáticos y extremos diferentes a los de su lugar de residencia donde la seguridad del visitante es responsabilidad de los actores locales del cantón. (Montesdeoca, Lemoine, Zamora, Zambrano, \& Álvarez, 2018)

Como hace referencia (Silberman, 2012)los atractivos turísticos se clasifican en naturales y culturales, (López, 2012)nos dice que los tractivos son el principal factor de atracción. Existen atractivos naturales y bienes creados construidos por la mano del hombre: culturales, históricos, religiosos, 
recreativos, contemporáneos, entre otros que hacen se jerarquizan de acuerdo a las actividades turísticas y del servicio que en el mismo se realizan.

En el cantón Sucre los atractivos turísticos son muy desconocidos por los turistas ya que no cuentan con una buena promoción, tampoco hay algún espacio adecuada para que el turista ya sea nacional como extranjero pueda pedir información sobre los diferentes atractivos que posee el cantón, dentro de este existen un sin número de atractivos unos atractivos naturales, como también bienes creados, una propuesta para dar a conocer los atractivos sería poner señaléticas para fomentar el desarrollo turístico.

La ciudad de Bahía de Caráquez denominada cuna de la nacionalidad ecuatoriana a su vez se le atribuye la denominación de "La ciudad sin copia" reconocida por pobladores, emigrantes y turista tanto nacional como internacional. Esta prestigiosa ciudad su principal atractivo son sus playas cautivadoras, divertidas, así como también su gastronomía sin dudar alguna una de las más exquisitas dentro de la provincia de Manabí, como no por supuesto de nuestro país y su gente bondadosa considerada una de las más tranquilas y seguras a nivel del balneario de la nacionalidad ecuatoriana.

Dentro de las variedades turísticas, podemos decir que posee recursos y atractivos turísticos que, aunque denotan acogidas por sus visitantes han venido careciendo de asistencia y de mantenimiento por los gobiernos locales, lo que ha traído consigo deterioro de estos y una imagen no acorde a las exigencias del mercado-

Estos sitios representativos poseen además un reconocimiento por sus paisajes que conjugan con un ambiente cultural y un entorno amigable que lo identifica con sus comunidades y habitantes entre estas las parroquias de San Jacinto y San Clemente cuya litoral playa tiene un reconocimiento por sus extensiones.

Cabe indicar que los efectos telúricos suscitado en el año 2016 dejaron pérdidas irreparables tanto a nivel de recursos como a nivel de infraestructuras. A partir de este evento a Extensión de Bahía de la Universidad Laica Eloy Alfaro de Manabí bajo la directiva del reconocido investigador doctor en ciencias Wilfredo Valls Figueroa direccionó actividades y proyectos alineados al desarrollo del turismo sostenible en la zona objeto estudio.

El principal inconveniente en el cantón Sucre es la deficiente identificación de los atractivos y recursos turísticos además de reconocer y tomar en cuenta la deficiente planificación territorial y turística que ha provocado que el acceso a los tractivos y recursos turísticos denoten una baja frecuencia de turistas nacionales e internacionales al destino. (Valls, Lemoine, Carvajal, \& Hernández, 2019)

Como objetivo esta identificar los diferentes atractivos que existen en el cantón para su respectiva potencialización con la finalidad de establecer estrategias para ayudar al desarrollo turístico del cantón Sucre y fortalecer así la actividad turística en la zona y los indicadores económicos del sector. 
Los actores turísticos conscientes de que es importante desarrollar acciones que hagan del turismo una actividad sostenible buscan estrategias sólidas para conservar los atractivos turísticos en la zona objeto estudios, aunque se prescinde de mejores técnicas de marketing para posicionar a los mismos (Lemoine, Navarrete, Valls, Carvajal, \& Villacis, 2018)

Estudios preliminares han concebido identificar las brechas pertinentes encaminados a desarrollar estrategias con enfoque sostenible. A partir de la creación del Observatorio Turístico en la Extensión de Bahía de Caráquez se direccionaron actividades de levantamientos y diagnósticos a nivel de actividades turísticas, de recursos y atractivos turísticos que aporte a la realización de inteligencia de mercados, además en la creación y gestión de un sistema de indicadores que resumidos en un cuadro de mando integral. (Carvajal et al, 2021)

(González \& González, 2021) desarrollan en su investigación una propuesta de indicadores para evaluar el turismo sostenible y poder lograr un destino económicamente activo partiendo de revisiones teóricas que le aportó a la toma de los indicadores que atributan a las especificaciones sociales y culturales del destino turístico, coincidiendo algunos de estos a los del Observatorio Turístico de Bahía de Caráquez.

Otros estudios relacionados a la temática de atractivos y recursos se han desarrollado como medio de potencializar otras actividades turísticas en la zona objeto estudio con la finalidad de buscar alternativas a los efectos provocado por la pandemia del Covid 19, como estrategias de marketing deportivo el destino Sucre-San Vicente (Lemoine, Hernández, Josselin, \& Kely, 2021)

\section{MÉTODOS Y TÉCNICAS}

El estudio tiene un carácter cualitativo porque a partir del método explicativo e ilustrativo se referencia a los atractivos turísticos como un potencial turístico que contribuye al desarrollo económico de las comunidades y las familias existentes en el cantón, además de incentivar a los actores a emprender e innovar nuevas fuentes de empleo logrando mejores de niveles de satisfacción de los turistas que frecuenten la localidad.

Desde la investigación cuantitativa se considera de acuerdo a los datos registrados por el ministerio de turismo y otras organizaciones he asentado datos del año 2015 sobre los turistas que visitan a Ecuador entre los extranjeros que más prenotan en el país, a la provincia Manabí se registran un total de turistas de un $6 \%$ el cual un 3\% llega al cantón Sucre donde su principal interés es conocer sus diferentes atractivos y recursos que posee la zona, como sus playas, centros de recreación, artesanías, gastronomía, costumbres, entre otros. 
El método analítico permitirá realizar una verificación a partir de cifras estadísticas existente en el Observatorio Turístico de la Extensión donde se reflejan de acuerdo a la demanda turística que llega al país, tal y como se muestra en la tabla 1.

Tabla 1: Nivel de representatividad turística.

\begin{tabular}{|c|c|c|}
\hline \multicolumn{3}{|c|}{ Afluencia de turistas } \\
\hline \multicolumn{3}{|c|}{ TURISMO NACIONAL } \\
\hline & $\begin{array}{|lc|}\text { NÚMERO } & \text { DE } \\
\text { HUESPEDES } & \\
\end{array}$ & $\%$ \\
\hline Manabí & 93627 & $6 \%$ \\
\hline Guayaquil & 234064 & $15 \%$ \\
\hline Quito & 652652 & $42 \%$ \\
\hline Otras prov. & 62417 & $4 \%$ \\
\hline \multicolumn{3}{|c|}{ Turismo internacional } \\
\hline Usa & 244.406 & $16 \%$ \\
\hline Colombia & 200.487 & $13 \%$ \\
\hline Canadá & 22.839 & $1 \%$ \\
\hline \multirow[t]{2}{*}{ Europa } & 49.937 & $3 \%$ \\
\hline & 1.560 .429 & $100 \%$ \\
\hline
\end{tabular}

Fuente. Autores, 2019

Como señala (Bernardez, 2011) recurso turístico son los elementos naturales, culturales, tradiciones, costumbres y acontecimientos que posee una determinada zona o área, con un potencial que podría captar el interés de los visitantes, pero a su vez (Alvarez, 2012) refiere que los recursos turísticos son la base sobre la cual se desarrolla la actividad turística y están constituidos por aquellos atractivos que, en un destino turístico, pueden generar el interés que influya en la elección del mismo y motivando el desplazamiento o la visita por parte del potencial consumidor.

Referente a las citas anteriores podemos recalcar que los recursos turísticos de un sitio son todas aquellas características, que hacen la diferencia de un lugar de otro sitio. Cada sitio turístico cuenta con recursos que lo identifican de acuerdo a su manifestación y que se le atribuye actividades histórico culturales propias del sector.

Dentro del cantón podemos decir que el impacto turístico que emblema la ciudad beneficia una gran variedad de atractivos por su fauna, flora y el paisajismo típico de la zona que a su vez involucran a la atracción de esta región haciendo que la existencia de estos atractivos por su diversidad y connotación.

La ciudad de Bahía de Caráquez reconocida como la ciudad ecológica por moradores de la zona y la ciudad patrimonial por gobiernos en tránsito que la identifican los turistas por su tranquilidad, hospitalidad y su seguridad, además por los sitios arqueológicos existentes. Poseedora de extensas playas 
para desarrollar deporte acuático, su paradisiaca Isla Corazón rodeada de manglares, crustáceos y aves endémicas en el estuario del rio Chone.

El turismo en el Ecuador constituye una actividad importante que ha ido creciendo en los últimos años. Varios son los atractivos que se destacan a la hora de hablar de nuestro cantón. Sin embargo, existen muchos otros que por falta de promoción no son lo suficientemente conocidos tanto a nivel nacional como internacional.

Para (Sigcho, 2013), hace referencia que para la realización de un inventario de atractivos turísticos se debe realizar los siguientes pasos:

$\checkmark$ Clasificación de los atractivos. -Consiste en identificar claramente la categoría, tipo y subtipo, al cual pertenece el atractivo a inventariar.

$\checkmark$ Recopilación de Información. - En esta fase se selecciona tentativamente los atractivos para lo cual se investigan sus características relevantes. Esta fase de investigación es documental, cuya información debe obtenerse en las oficinas relacionadas con su manejo.

$\checkmark$ Trabajo de Campo. -Consiste en la visita a efectuarse a los sitios para verificar la información sobre cada atractivo. Es el procedimiento mediante el cual se le asignan las características al atractivo.

$\checkmark$ Evaluación y Jerarquización

Como hace referencia (MINCETUR, 2006) referente al levantamiento del inventario de recursos turísticos comprende dos fases de trabajo:

Fase I.- Ordenar y clasificar los lugares, objetos, acontecimientos y otros de interés turístico de los recursos turísticos del país, región o área determinada. Proceso que se refiere a la recopilación de información, ordenamiento y clasificación de los datos.

Fase II.- Evaluación y jerarquización de los recursos turísticos, que comprende el proceso de ponderación de los recursos, con el fin de determinar su grado de importancia.

De esta manera podemos decir que la jerarquización nos permite conocer de mejor manera cada atractivo al que deseamos llegar y de este modo promocionar el destino con las herramientas adecuadas para obtener una mayor demanda y así beneficiar tanto al cantón y sus pobladores. 


\section{POBLACIÓN}

Para el desarrollo del estudio referente al diagnóstico del estado de conservación de los atractivos y recursos turísticos se tomará en cuenta el total de la población del último censo realizada en el 2010. Con una estimación de 57.159 Habitantes según se refleja en el estadístico mostrado a continuación:

Tabla 2. Estadístico poblacional del cantón Sucre

\begin{tabular}{|l|l|l|l|}
\hline PARROQUIAS & $\mathbf{N}^{\mathbf{0}}$ POBLACION & \%POBLACION & MUESTRA \\
\hline Bahía & 26.112 & $45.68 \%$ & 54 \\
\hline Charapotó & 20.060 & $35.09 \%$ & 41 \\
\hline San Isidro & 10.987 & $19.22 \%$ & 23 \\
\hline TOTAL & $\mathbf{5 7 . 1 5 9}$ & $\mathbf{1 0 0 \%}$ & $\mathbf{1 1 8}$ \\
\hline
\end{tabular}

Fuente: Autores, 2020

La muestra es de 118 habitantes divididos en 54 habitantes en la parroquia de Bahía de Caráquez, 41 habitantes en la Parroquia de Charapotó y 23 habitantes en la Parroquia de San Isidro con una confiabilidad de $95 \%$ y un margen de error del $9 \%$.

\section{RESULTADOS}

En el último registro realizado en el año 2016 por el ministerio de turismo en el cantón Sucre se obtuvo la existencia de treces atractivos en la parroquia de Bahía de Caráquez, ciudad cabecera cantonal e insignia del cantón.

Tabla 3. Levantamiento de atractivos en el cantón Sucre.

\begin{tabular}{|l|l|}
\hline 1. Museo Bahía de Caráquez & 8. Casa Patrimonial \\
\hline 2. Reserva Biológica Cerro Seco & 9. Parque Saiananda \\
\hline 3. Playa Bahía de Caráquez "Paseo Roberto" & 10. Parque Ecológico y Arqueológico Chirije \\
\hline 4. Mirador "La Cruz" & 11. Playas Pajonal \\
\hline 5. Refugio de Vida Silvestre "Isla Corazón" & 12. Manglar "La Boca" \\
\hline 6.Mirador Bella Vista & 13. Iglesias "Las Mercedes" \\
\hline 7. Playa Punta Bellaca & \\
\hline
\end{tabular}

Fuente: Autores, 2020

A través de técnica de observación se realizó una evaluación de los treces atractivo ubicado en las comunidades perteneciente a Bahía de Caráquez para medir estado de conservación, acceso y actividades turísticas vigentes, declarándole en colapso total, parcial o activo. En el siguiente cuadro se reflejan lo que tienen daños parciales y los que están activos. 
Tabla 4. Identificación de atractivos turísticos.

\begin{tabular}{|l|l|l|}
\hline$N^{o}$ & ATRACTIVOS TURISTICOS & IDENTIFICACIÓN \\
\hline 1 & Museo Bahía de Caráquez (colapso parcial) & Cultural y Arqueológico \\
\hline 2 & Reserva Biológica "Cerro Seco" & Bosque protector de flora y aves endémica \\
\hline 3 & Playa Bahía de Caráquez "Paseo Roberto" & Playas y especies de vida silvestre \\
\hline 4 & Mirador "La Cruz" (colapso parcial) & Paisajismos culturales \\
\hline 5 & Isla Corazón & Refugio de vida silvestre, fauna y flora \\
\hline 6 & Playa "Punta Bellaca" & Playa y vida silvestre \\
\hline 7 & Parque Saiananda & Parque ecológico y reserva de aves nativas \\
\hline 8 & Parque Chirije & Ecología y arqueología \\
\hline 9 & Playas Pajonal & Playas y paisajismo natural \\
\hline 10 & Manglar "La Boca" & Bosque pantanoso y ecosistema diverso. \\
\hline 11 & Iglesia" La Merced" & Histórico cultural \\
\hline
\end{tabular}

Fuente: Autores, 2020

\subsection{CARACTERIZACIÓN DE ATRACTIVOS EN LA PARROQUIA BAHÍA DE CARÁQUEZ}

\subsubsection{Museo Arqueológico Bahía de Caráquez}

En nuestra localidad poseemos uno de los museos más emblemático de nuestro país ya que conserva piezas de nuestra cultura "Caras, Jama Coaques", de nuestros antepasados que vinieron en balsas con grupos de nativos que se asentaron en la parte norte de Manabí, internamente en el museo tiene fragmentos arqueológicos de las diferentes épocas sobre el tipo de vida que llevaban, como las diferentes culturas Machalilla, Jama Coaque y la Manteña

Está ubicado en el centro de la ciudad en el malecón Alberto F. Santos, cuenta con cinco pisos, tres pisos de exhiben las diferentes arqueologías y artes visuales. Cuentan con personal altamente calificado, en varios tipos de idiomas que hace que la visita tanto local como extranjera sea agradable. En la actualidad se encuentra en restauración.

Figura 1. Museo arqueológico






\subsubsection{Reserva Biológica Cerro Seco}

Según (Viajandox, 2015) argumenta que la Reserva Biológica Cerro Seco, se encuentra frente al Océano Pacífico, al sur oeste de la ciudad ecológica ("eco city") Bahía de Caráquez, en la provincia de Manabí, en el Ecuador. El bosque de la reserva es tropical muy seco tropical, deciduo por la sequía con 500 a 1000 milímetros de lluvia por año y dos estaciones muy marcadas, con precipitaciones desde fines de diciembre hasta inicios de abril.

Correspondiente a la cita mencionada anteriormente podemos indicar que este lugar contiene una diversidad de enorme flora endémica entre las más principales especies están palo santo, moyuyo, guayacán entre otros. No obstante, también posee considerables números de aves que solo pueden ser observados en las mañanas, esta reserva se encuentra protegida por las personas que habitan en el lugar, cerro seco brinda posibilidad de trabajo como también proyectos de investigación.

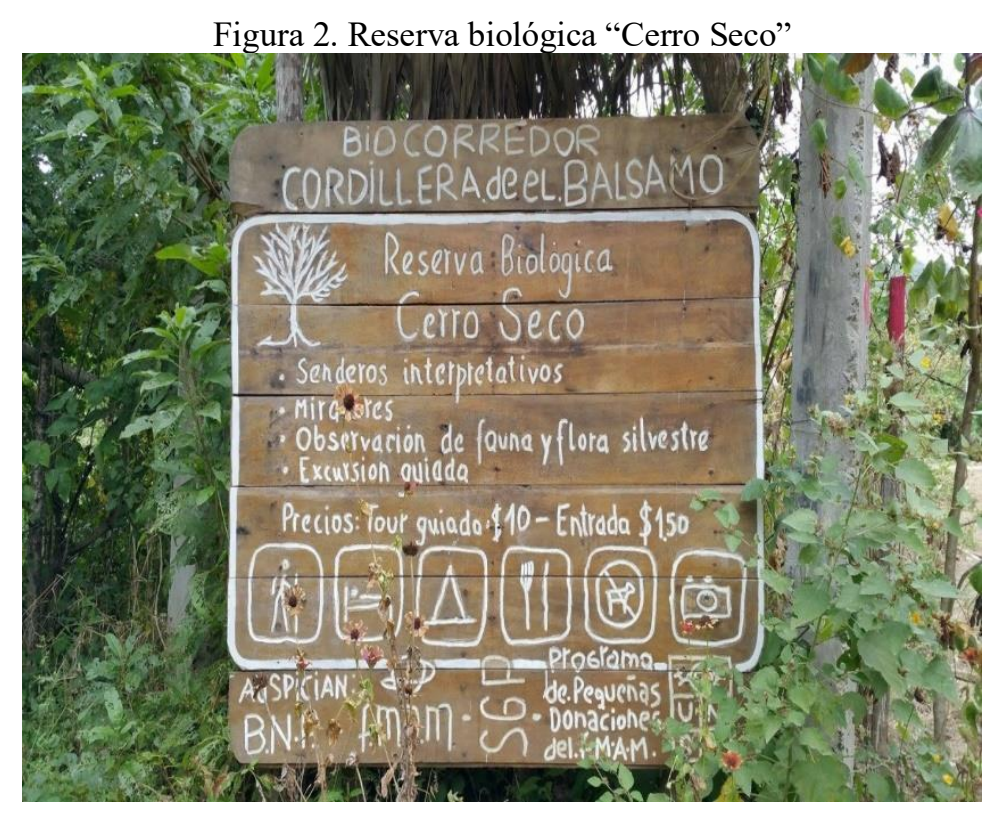

\subsubsection{Playa Bahía de Caráquez}

Según (Turismo aventura playa y surf en Bahía de Caraquez, 2012) En la playa de Bahía de Caráquez se puede practicar un sin número de deportes acuáticos, entre ellos están: el surf, kayak, windsurfing y el famoso esquí acuático.

En la ciudad de Bahía de Caráquez privilegiada por estar en la zona costera posee una de las playas encantadoras, atractivas y muy tranquilas de su sector acoplando en si la vista hacia el cantón San Vicente esta playa colinda con la desembocadura del rio Chone existen actividades de recorridos y paseos en botes y motos acuáticas. 
Esta playa tiene una extensión aproximada de 1400 metros los cuales 600 metros que le pertenecen a la playa frente al estuario del rio sin olas, y del otro costado los 800 metros que se encuentra al frente del océano pacifico en donde se pueden evidenciar la presencia de olas y puesta del sol.

\section{Figura 2. Vista panorámica de la playa "Paseo Roberto"}

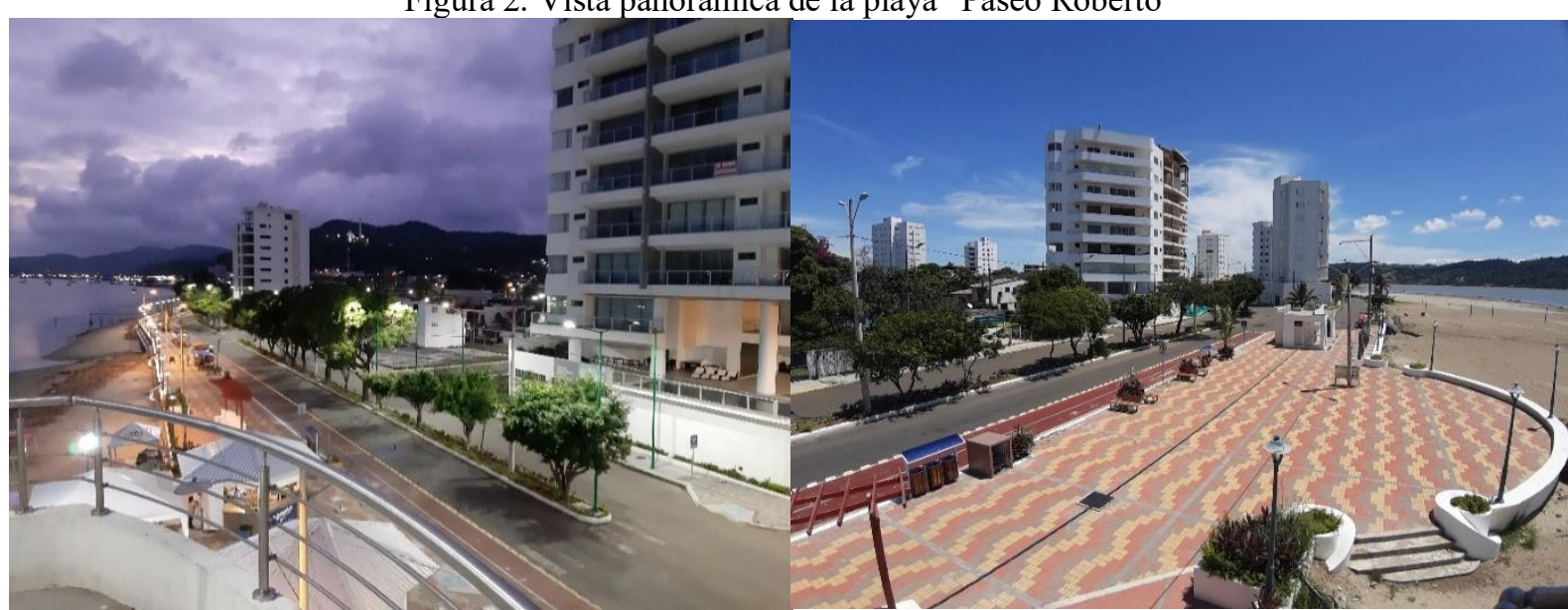

\subsection{MIRADOR “LA CRUZ”}

Una cruz de concreto de 19 metros de alto que se levanta en una colina es el nuevo atractivo turístico de Bahía de Caráquez, en el Cantón Sucre.

Este mirador en donde podemos divisar el paisaje de la ciudad de Bahía de Caráquez, el estuario del rio Chone y las entrantes de mar del océano pacifico podemos decir que es uno de los atrayentes emblemáticos del sector, que son visitados en los días de descanso, en su alrededor tenemos áreas infantiles y habitantes del lugar que se dedican a la venta de refrigerios y bebidas, ya que es un ingreso económico que los ayudan a tener una mejor calidad de vida. En la actualidad se encuentra abandonado en fase de remodelación.

En este sitio es de muy fácil acceso que se puede ingresar por tres trayectos una por las escalinatas que se encuentran situada en el barrio Pedro Fermín Cevallos, otra mediante vehículo ingresando por el barrio María Auxiliadora, y; por otra vía de acceso es por la parroquia Leónidas plaza del barrio reales tamarindo.

Estudio de afluencia turísticas en el destino muestran que las visitas al mismo representan el 71.5\% de los turistas que frecuentan el destino, de estos el $15 \%$ es turista internacional y el $56.5 \%$ representado por turismo local, regional y nacional. 


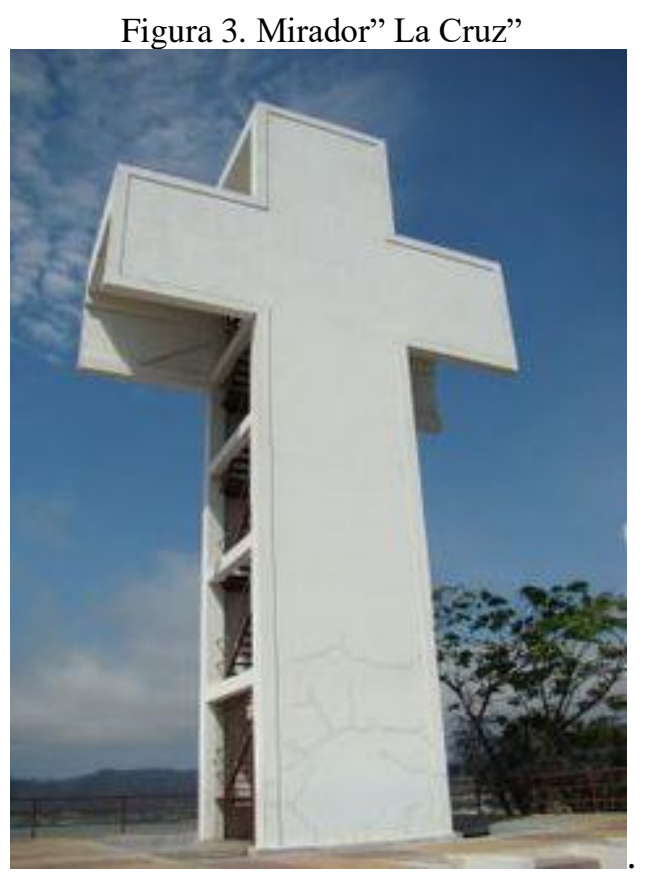

\subsubsection{Isla Corazón}

Según (Ministerio del ambiente, s.f.) El RVS Isla Corazón y Fragatas es uno de los productos estrellas de la Ruta del Spondylus de la zona norte de Manabí (Bahía de Caráquez y San Vicente) desde el año 2010 hasta el 2015 se han registrados 22.794 turistas entre nacionales y extranjeros. El ecosistema manglar y la gran biodiversidad que estos albergan; ofrece oportunidades para el desarrollo de actividades turísticas donde las comunidades son participes de la experiencia de los turistas en esta área.El refugio cuenta con un área aproximada de 50 hectáreas en la cual en su interior hallamos gran variedad de aves fragatas las cuales son más de 60 ejemplares de aves entre nativas, migratorias y congregarías del Pacifico Sudamericano que se encuentran dentro de la isla.

Según (MINTUR, 2013) el refugio de vida silvestre Isla Corazón y Fragatas es una reserva natural que protege dos islas con 800 hectáreas de manglares, las islas principales son la "Isla Corazón, Fragatas y la Isla del Sol”. Los atractivos más interesantes son los túneles que recorren toda la Isla Corazón por debajo del ramaje de manglar, donde podremos a bordo de una canoa observar la biodiversidad de flora y fauna que produce la isla, además se encuentra un corredor o sendero de madera que permite el ingreso al interior del manglar por medio de una corta caminata de 400 metros donde el turista podrá experimentar un ambiente único natural.

La reserva ofrece su acceso de manera posible a sus diferentes senderos y mirador los comuneros del sitio quienes conservan el lugar y los guías del área se encuentran ubicada en la desembocadura del rio Chone donde unen las aguas del océano pacífico y el agua dulce del rio estas dos aguas ayudan a la formación del bosque del manglar, según se muestra en la figura 4. 
Figura 4. Atractivo refugio de vida silvestre Isla Corazón y Fragatas
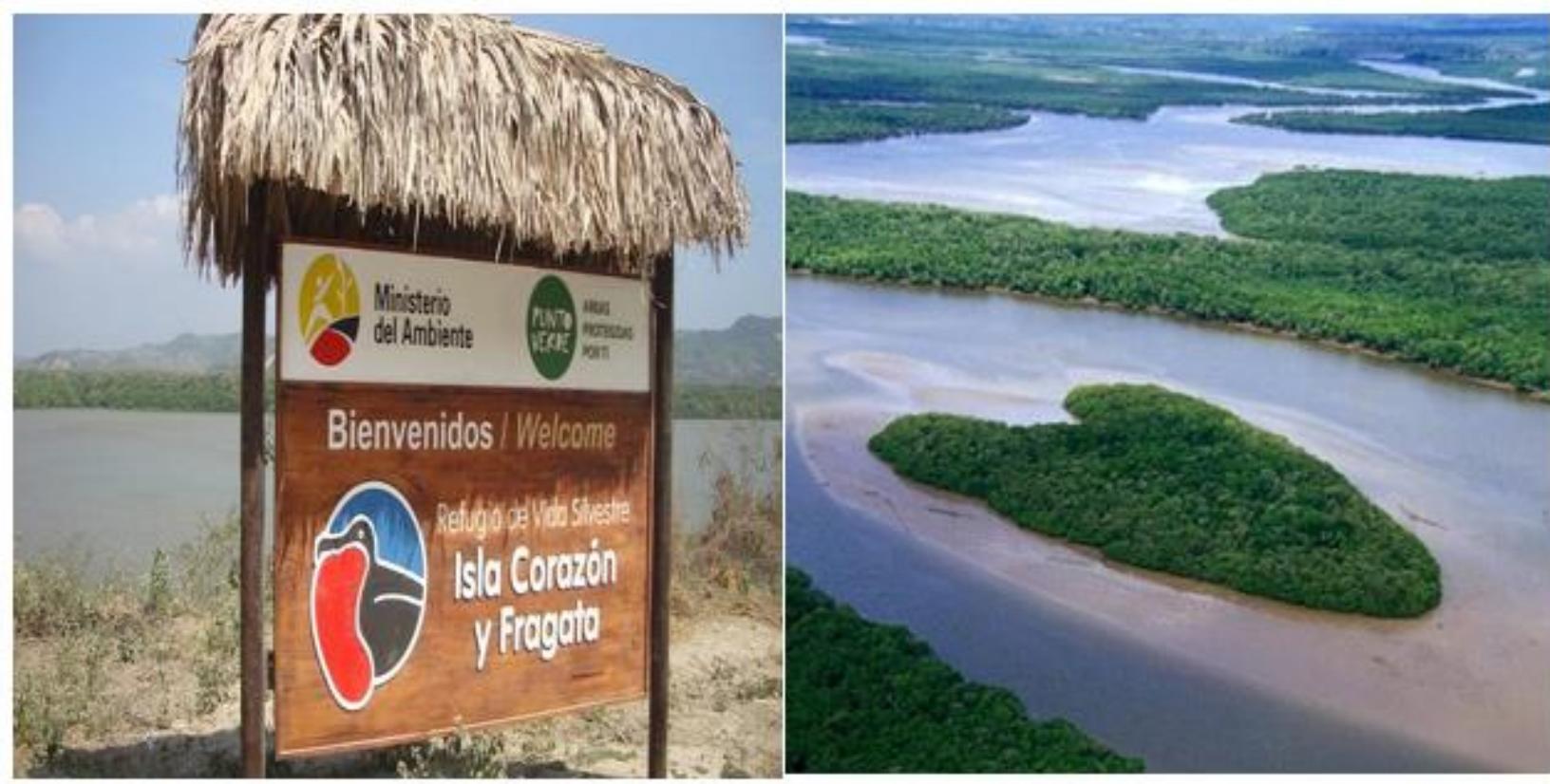

Más de 60 especies de aves entre nativas, migratorias han sido identificadas dentro de la isla durante las diferentes épocas del año, pero entre ellas las más comunes están ibis, garzas nocturnas garzas, garzas níveas, garzas reales, garzas bulleras, cormoranes, tórtolas, garcillas estriadas, mariposas, murciélago blanco, martín pescador grande y martín pescador verde, zarapitos trinadores, agujetas, entre otros. También se encuentran otras especies como iguanas, la boa constrictor, y una amplia variedad de crustáceos, moluscos y curiosos insectos. Allí se encuentra una de las colonias más grandes de aves fragatas del Pacífico. Esta colonia se triplicó en los últimos 8 años, gracias a la reforestación y rescate del manglar, así como otras especies endémicas que a programas de vida silvestre se han rescatado.

\subsection{ATRACTIVOS DE LA PARROQUIA LEONIDAS PLAZA}

\subsubsection{Playa Punta Bellaca}

Como nos dice (Navia, 2015) Paradisíaca y extraordinario balneario ubicado a apenas 5 minutos de Bahía de Caráquez. Es una playa ideal para permanecer en familia para un día de camping playero. Se trata de una playa alternativa para personas que prefieren el descanso y la tranquilidad. Para poder acceder a esta playa se ingresa por la vía de la ciudadela "Jorge Lomas", donde se observa grandes extensiones de playas que tiene aproximadamente $4 \mathrm{~km}$ colindando con las playas más cercanas como La mesita, Punta Gorda, La gringa, y El bejuco en este lugar se puede apreciar un sin número de aves, entre los más comunes: pelicanos, piqueros, gaviotas, fragatas, cangrejos entre otros, dentro de este bello horizonte 
podemos encontrar algas y en las colinas de este lugar se encontraran varios tipos de árboles entre las comunes del sector monte salado, cactus, pitajaya, ceibo y varias especies más.

En este lugar inclusive en su paisaje también, las personas de su localidad se dedican a la pesca, así como también van en la búsqueda de ostras marinas que son muy apetecidas en nuestros balnearios en las comidas típicas de la costa siendo de estas un ingreso económico para las personas que se dedican a este trabajo, también en las épocas de temporada playera asisten turistas nacionales e internacionales donde hay juegos deportivos y acuáticos. Este recurso natural se encuentra en la espera de desarrollo de productos turísticos para potencializar la actividad deportiva o recreativa o para desarrollar turismo de aventura.

Figura 5. Playa Punta Bellaca

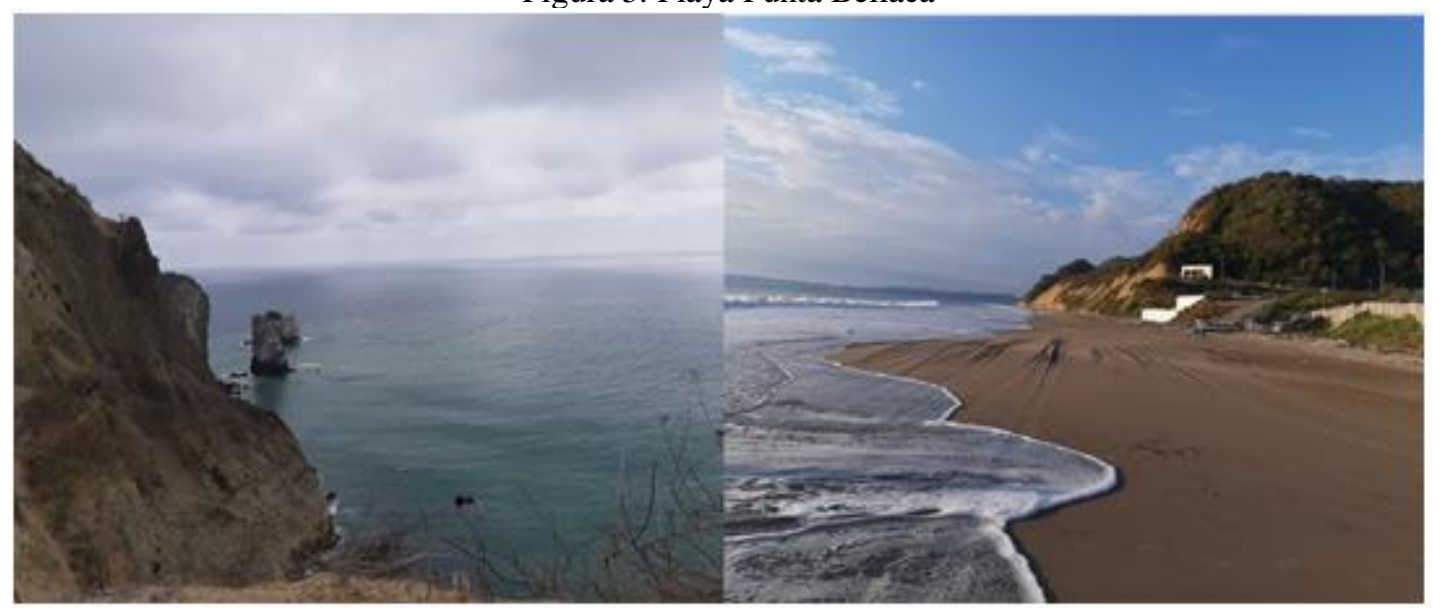

\subsubsection{Parque Sainanda}

Como hace mención (Hora, 2004) Saiananda nació como un proyecto turístico, pero jamás pensé en ganar dinero. Quienes más visitan el parque son escolares", comenta Alfredo Harmsen.

El parque se encuentra ubicado en la vía km8 antes de llegar al sitio papilandia, al costado de la carretera principal, en este parque las visitas que realizan tanto de los habitantes de la zona como también personas extranjeras es un lugar muy exótico en donde podemos apreciar varios senderos y en el trayecto de la visita correspondiente se pueden apreciar platas exóticas animales de campo como también en la subida a la loma se puede apreciar la vista al estuario rio Chone, dentro de esta reserva que es un pequeño sitio al cuidado donde brindan amor y se alojan muchas especies de la flora y la fauna entre ellos podemos decir que algunas animales sufren golpes, heridas y las personas encargadas de este lugar se encargan de brindar su apoyo al cuidado y esmero de este lugar, que es muy pacifico donde abundan la extrema naturaleza.

Este lugar brinda sitio de hospedaje brindando zonas de descanso, restaurant y todo lo que concierne a la experiencia de la naturaleza también en el trayecto de la excursión se puede pasar por debajo 
de la carretera hacia el otro costado del parque en donde tienen bajo su cuidado varios tipos de especie de aves como palomas, codornices, pavos reales, patos, loros, pericos.



\subsubsection{Parque Ecológico y Arqueológico Chirije}

Como hace mención el periódico (La Hora, 2010) el arqueólogo guayaquileño Emilio Estrada descubrió el sitio en la década de 1950, y nombró una nueva cultura de la llamada Chirije. De sus investigaciones se dedujo que fue un antiguo puerto marítimo, de la cultura Bahía.

Este parque contiene 238 hectáreas de un bosque tropical seco, lo podemos encontrar durante unos 20 minutes de viaje desde la ciudad de Bahía de Caráquez mediante un divertido recorrido en chiva dando en si la maravilla del deleite de la música tropical en el trayecto a este sitio. En la actualidad se encuentra abandonado luego d ellos sucesos telúricos del 2016.

Considerando que el parque es uno de los más nuevos de la costa ecuatoriana, cuenta como un sitio muy importante dentro de los sitios arqueológicos del país, dentro del parque podemos estar a la mira de aves como periquitos del pacifico, insectos de palo, reptiles y un sinfín de mariposas de colores, cuenta también una gran variedad de ceibos y otras clases de árboles muy conocidas del sector entre ellos guayacán, palo santo, moyuyo, laurel muy típica en los bosques secos, podemos recorrer los senderos muy apropiadamente señalados, recorridos a caballo, visitas en el museo del sitio donde podemos encontrar excavaciones de tesoros precolombinos.

A partir de allí, al menos una veintena de arqueólogos nacionales y foráneos descubrieron piezas que se consideran faltantes de la antigüedad de la Costa, localizando vestigios de cerámica, conchas, huesos y restos de las culturas Bahía, Jama Coaque, Chirije y Manteño (500 AC - 1534 DC) 


\subsubsection{Playas del Pajonal}

Según (Rodriguez, 2015) relata como si esta playa estuviera escondida, a 15 minutos de camino por la playa de Bahía de Caráquez (Manabí), está Chirije. Es un sitio lleno de historia, supersticiones y naturaleza. Con un clima húmedo, debido a que se encuentra rodeado de la Reserva Biológica Cerro Seco, Chirije fue una localidad económica importante en la Cultura Bahía (500 a.C. - 500 d.C.), en especial por su actividad portuaria. Todo el ambiente de Chirije tiene un aroma a historia y si tiene una característica en particular, ese es su silencio. Fue redescubierto en la década del 50 por el arqueólogo Emilio Estrada.

En esta playa que se encuentra ubicada a $18 \mathrm{~km}$ de la ciudad Bahía de Caráquez es una de las más visitadas del sector caracterizado al ser un lugar muy tranquilo, relajador perfecto para acampar y por tener un paisaje esplendoroso podemos también apreciar la visita de las ballenas jorobadas que vienen por temporada en los meses de junio y septiembre, las cuales son un entretenimiento a los turistas tanto nacionales y extranjeros, en la actualidad se encuentra abandonada.

Tiene una proximidad de 1.5 kilómetros de playa su clima es muy variable bordando entre los $25^{\circ}$ c y $29^{\circ} \mathrm{c}$ en este lugar también podemos apreciar una gran variedad de aves tales como: fragatas, pelicanos, entre otras aves migratorias y endémicas.

\subsection{RECURSOS TURÍSTICOS DE BAHÍA DE CARÁQUEZ}

Los recursos turísticos son lugares capaces de atraer visitantes y son la base del desarrollo de la zona los recursos existen en todas partes, pero lo importantes es que sean explotados. Hago referencia de los primordiales recursos del cantón.

\subsubsection{Isla de "Las Fragatas"}

Su calificativo se debe a que en el lugar predominan las aves fragatas, llamadas también piratas. Estas aves son muy sensibles al ruido y voces humanas, expresan su enojo expulsando el alimento que guardan para sus crías, El suelo de la isla está constituido de lodo y arena, encontrándose almejas, conchas, mejillones la Isla de las Fragatas se encuentra ubicada en el estuario del río Chone a solo 30 minutos de Bahía de Caráquez.

\subsubsection{Estuario Rio Chone}

El estuario se encuentra ubicado en el cantón sucre en el sector de la parroquia Leónidas plaza, tiene una magnitud de $36 \mathrm{~km}$, este maravilloso estuario puede ser observado desde los cerros de Mauricio. En él se pueden hacer recorridos en lancha donde se pueden observar una dimensión de manglar que se encuentra ubicado en las islas existentes dentro del estuario, donde también podemos observar la isla de 
los pájaros, este estuario cuenta con una variedad de vegetación podemos indicar algunas: algarrobos, moyuyo, monte salado.

\subsubsection{Iglesia "La Merced"}

Esta iglesia es de estilo historicista, ya que usan formas, elementos y detalles de arquitectura de épocas pasadas, arcos ojivales y aculo gótico. La cubierta tiene una pronunciada inclinación y teatinas, lucerna-rías y torre de reloj con capital piramidal y cornisa periférica de cubierta, en la estructura vista de la cubierta se utiliza par y nudillo modificado.

La Iglesia La Merced se encuentra en el cantón Sucre, en la parroquia Bahía de Caráquez, Su estilo original se conserva por cuanto la iglesia tiene mantenimiento, lo que ha permitido su permanencia, es de origen muy antiguo en la región por sus doctrinas se construye en la ciudad de Bahía de Caráquez desde 1906 hasta 1992.

\subsubsection{Isla de los pájaros}

La isla de los pájaros es una pequeña isla frente al estuario del rio Chone que posee gran variedad de especies de ave entre ellas: pelicanos, garzas, patillo entre otros. Este sitio no es tan conocido pero este lugar es extraordinario para el turismo ecológico, recreativo de aventura.

En este lugar el principal atrayente es la variedad de aves aquí el turista podrá disfrutar de sus aguas tranquilas y cristalinas, que aran que el viajero se sienta con la confianza de disfrutar sin ningún riesgo, en el lugar se puede recolectar almejas, cangrejos en este sitio se puede realizar la pesca artesanal ya sea mediante atarraya o trasmallo.

\begin{tabular}{|l|l|c|l|}
\hline $\mathbf{N}^{\circ}$ & RECURSOS & ACTIVIDADES & $\%$ \\
\hline $\mathbf{1}$ & Isla de las Fragatas & 5 & $22 \%$ \\
\hline $\mathbf{2}$ & Estuario de Chone & 3 & $13 \%$ \\
\hline $\mathbf{3}$ & Mirador bellavista & 4 & $17 \%$ \\
\hline $\mathbf{4}$ & Isla de los pájaros & 6 & $26 \%$ \\
\hline $\mathbf{5}$ & Iglesia la Merced & 5 & $22 \%$ \\
\cline { 2 - 3 } & TOTAL & 23 & \\
\cline { 2 - 3 } & \multicolumn{2}{|c|}{ Fuentes: Autores, 2020 } \\
\end{tabular}

Por tipo de actividad el atractivo mejor evaluado fue la Isla de los Pájaros que represento un $26 \%$ por tener 6 tipos de actividades turísticas reconocido dentro de este atractivo, seguido por la Iglesia la Merced que tiene 5 actividades de carácter turísticos que ayudan y fomentan al turismo en la zona al igual que la Isla de las Fragatas que a pesar de tener 5 actividades es un atractivo de concurrencia de turismo 
internacional según fuentes estadísticas de registro de visitantes. En la actualidad todos se han visto afectado por el Covid 19 y se trabaja en la proyección estratégicas de reactivación de las actividades en las medidas establecidas por el gobierno en turno bajo las condiciones pandémicas.

\section{CONCLUSIONES}

Entre las conclusiones más explicativas que puedo destacar son las siguientes

- La caracterización de atractivos y recursos turísticos permitió recopilar información de los diferentes atractivos que posee el cantón para evaluar estado y conservación de estos y poder definir estrategias efectivas.

- El estudio permitió evidenciar la potencialidad de los atractivos y recursos turísticos para desarrollar actividades turísticas partiendo de la estacionalidad de la demanda existente en el cantón para mercados emisores como Canadá, China, Italia entre otros del área latinoamericano.

- Se demostró en el estudio que el cantón Sucre tiene potencial turístico caracterizado por el paisajismo, la flora y la fauna que le aporta a los atractivos y recursos turísticos interesantes actividades recreativas, de esparcimiento y entretenimientos que incidirán en un crecimiento de flujo de turista al destino. 


\section{BIBLIOGRAFÍA}

Alvarez, E. A. (2012). Los recursos turisticos.

Auquilla, V. R. (2012). "Estudio para la implementación de atractivos turísticos culturales en el barranco de cuenca". Cuenca, Ecuador.: Universidad de Cuenca facultad de Ciencias de la Hospitalidad Escuela de Turismo.

Bernardez, T. (2011). Slideplayer. Recuperado el 14 de agosto de 2016, de slideplayer: http://slideplayer.es/slide/4041300/

Bote, V. (1990). Planificación económica del turismo. México: Editorial Trillas. .

Cabezas, C. A. (2012). Diseño de un sistema de rutas turísticas en la parroquia salinas de Tomabela, cantón Guaranda, provincia de Bolívar. Riobamba, Ecuador.: Escuela Superior Politécnica de Chimborazo Facultad de Recursos Naturales Escuela de Ingeniería En Ecoturismo.

Carvajal et al. (2021). ontrol de la gestión del destino turístico Sucre-San Vicente -Jama-Pedernales, caso observatorio turístico. South Florida Journal of Development,v.2, n.2, p.2451-2461.

Cerro, F. L. (2015). Los Recursos Tuísticos en un Proceso de Planificación: Inventario y Evaluación. Consejo Superior de Investigaciones Científicas.

Corbuse-turismo. ( 2011). Recuperado el martes 09 de agosto de 2016, de Corbuse-turismo: http://corbuseturismo.blogspot.com/2011/07/clasificacion-de-atractivos-turisticos.html

Departamento de Turismo, G. A. (2016). Inventario de Atractivos Turisticos del Cantón San Vicente. Diario, E. (2007). Manglar de La Boca de Crucita ya tiene ordenanza. El diario.

Gobierno Autonomo Descentralizado del Cantón San Vicente. (s.f.). Obtenido de www.sanvicente.gob.ec González, A., \& González, B. (2021). Propuesta de indicadores para evaluar el turismosostenible. CantónOtavalo. South Florida Journal of Development,v.2, n.3, p.4239-4263.

Hora, L. (domingo 5 de diciembre de 2004). Saiananda. La Hora.

Isidro, G. R. (2011). Recuperado el agosto de 2016, de http://gpsanisidro.gob.ec/carchi/?paged=3

La Hora. (martes 12 de enero de 2010). Título. Vista de Chirije y la costa. Un paraíso escondido a 90 kilómetros al sur de la línea equinoccial. La Hora.

Lemoine, F., Hernández, N., Josselin, C., \& Kely, Z. (2021). Estrategias de marketing deportivo en tiempo de emergencia pandemica por el covid 19 en el destino Sucre - San Vicente.Ecuador, 2021. South Florida Journal of Development, p.1552-1564.

Lemoine, F., Navarrete, S., Valls, W., Carvajal, G., \& Villacis, L. (2018). Percepción de actores locales sobre turismo sostenible en comunidades de Leonidas Plaza del cantón Sucre, ManabÍ. Revista ECA Sinergia, Vol. 9 No2, pp.55-67. 
Llautong, J. L. (2013). "Evaluación del uso turístico y recreatvo de olón en la provincia de Santa Elena”. Guayaquil, Ecuador.: Escuela Superior Politécnica del Litoral Facultad de Ingeniería Marítima, Ciencias Biológicas, Oceánicas y Recursos Naturales.

López, H. H. (2012). Inventario Turistico. Recuperado el miercoles 11 de agosto de 2016, de Inventario Turistico: http://inventarioturisticolopez.blogspot.com/2012/12/inventario-turistico.html?view=classic Lucio Galiano Luna. (2016). Investigación Turística. Cusco, Perú.

MINCETUR. (2006). Dirección Nacional de Desarrollo Turístico,Dirección de Desarrollo del Producto Turístico . Ecuador.

Ministerio de Turismo. (2004). Scribd. Obtenido de Scribd: https://es.scribd.com/doc/56382986/Metodologia-de-Inventario

MINISTERIO DE TURISMO. (2007). Diseño del Plan Estratégico de Desarrollode Turismo Sostenible para Ecuador "Plandetur 2020". Ecuador.

Ministerio del ambiente. (s.f.). Recuperado el 23 de agosto de 2016, de http://www.ambiente.gob.ec/refugio-de-vida-silvestre-isla-corazon-y-fragatas/

MINTUR. ( 2007). Ministerio Nacional de Turismo. Recuperado el 29 de Julio de 2016, de Ministerio Nacional de Turismo: http://www.turismo.gob.ec/wpcontent/uploads/downloads/2013/02/PLANDETUR-2020.pdf

MINTUR. (2013). REFUGIO DE VIDA SILVESTRE ISLA CORAZÓN Y FRAGATAS. Recuperado el 23 de agosto de 2016, de https://www.ambiente.gob.ec/refugio-de-vida-silvestre-isla-corazon-y-fragatas/ Montesdeoca, M. G., Lemoine, F. Á., Zamora, Y. A., Zambrano, J., \& Álvarez, M. (2018). La seguridad turística e impacto social en comunidades del cantón San Vicente: caso playa "los perales". Mikarimin. Revista Científica Multidisciplinaria., pp.35-48.

Navia, C. (2015). Keep Calm Heart Manabì. Recuperado el 28 de agosto de 2016, de KEEP CALM HEART MANABİ: http://cesarnaviar.blogspot.com/2015/03/playa-punta-bellaca-manabi.html

Playas de Ecuador . (2016). Obtenido de playas de ecuador : http://www.playasdecuador.com/nuestracosta/costa-norte-ecuador.html

Rivera, S. C. (2009). Estudios del turismo . Recuperado el martes 9 de agosto de 2016, de Estudios del turismo: http://estudiosdelturismo.blogspot.com/2009/05/conceptos-de-patrimonio-atractivo.html

Rivera, S. R. (2008). BlogSpot. Obtenido de BlogSpot: http://estudiosdelturismo.blogspot.com/2009/05/conceptos-de-patrimonio-atractivo.htm

Rivera, S. R. (2009). BlogSpot. Recuperado el 27 de Julio de 2016, de BlogSpot: http://estudiosdelturismo.blogspot.com/2009/05/conceptos-de-patrimonio-atractivo.html

Rodriguez, M. (2015). Es posible llegar caminando por la playa cuando la marea está baja. El telegrafo. Sánchez, R. A. (2010). Módulo: Recursos Turisticos. Bloque I. Madrid: Liber Factory. 
Sigcho, K. S. (2013). "Diagnóstico situacional turístico de la parroquia rural manú del cantón saraguro de la provincia de Loja". Loja, Ecuador.: Universidad Nacional de Loja carrera de ingeniería en: "Administración Turística".

Silberman, A. G. ( 2012). prepaturismocorbuse2012. Recuperado el miercoles 10 de agosto de 2016, de prepaturismocorbuse2012: https://prepaturismocorbuse2012.wordpress.com/

Travel, e. (s.f.). google. Recuperado el 23 de agosto de 2016, de ggogle: http://www.ecostravel.com/ecuador/hoteles/manabi/playa-bahia-de-caraquez.php

Turismo aventura playa y surf en Bahía de Caraquez. (2012). Ecuado-turismo.com. Recuperado el 2016 de agosto de 2016, de http://www.ecuador-turistico.com/2012/08/turismo-aventura-playa-y-surf-enbahia-de-caraquez.html

Turisteandoecuador. (2015). Recuperado el 11 de agosto de 2016, de turisteandoecuador: https://turisteandoecuador.wordpress.com/2015/08/04/atractivos-turisticos-del-canton-sucre-en-manabi/ Valls, W., Lemoine, F. Á., Carvajal, G. V., \& Hernández, N. R. (2019). Identificación de atractivos turísticos de interés para el desarrollo del sector hotelero en los cantones de Sucre, San Vicente, Jama y Pedernales (Ecuador). REVISTA INTERNACIONAL DE TURISMO, EMPRESA Y TERRITORIO.N ${ }^{\circ}$ 6, pp. 113-130.

Viajando por Ecuador. (s.f.). Recuperado el 24 de agosto de 2016, de http://www.viajandox.com/manabi/mirador-santa-teresa-sucre.htm

Viajandox. (2015). Reserva Biológica Cerro Seco. Recuperado el 24 de agosto de 2016, de https://ec.viajandox.com/sucre/reserva-biologica-cerro-seco-A2997 